\title{
New Zealand Black Rabbit
}

National Cancer Institute

\section{Source}

National Cancer Institute. New Zealand Black Rabbit. NCI Thesaurus. Code C77109.

A New Zealand rabbit with black coat color and coat genotype Si, VV, E, D, C, B. The New Zealand black is commonly utilized in biomedical research in a variety of studies including developmental toxicity studies, toxicology studies, asthma research, and antibody production. 